\title{
Numerical Analysis of SHB Effects in Laterally- Coupled Distributed Feedback (LC-DFB) Lasers
}

\author{
Mahmood Seifouri \\ Shahid Rajaee Teacher Training University, \\ Faculty of ECE, \\ Lavizan, Tehran, Iran \\ mahmood.seifouri@srttu.edu
}

\author{
Fatemeh Shahshahani \\ Alzahra University, \\ Faculty of Science \\ Vanak, Tehran, Iran \\ f.shahshahani@alzahra.ac.ir
}

\author{
Ata Faraji \\ Shahid Rajaee Teacher Training University, \\ Faculty of ECE, \\ Lavizan, Tehran, Iran \\ airin_208@yahoo.com
}

\begin{abstract}
In this paper the stability of Laterally-Coupled Distributed Feedback (LC-DFB) Lasers against the Spatial Hole Burning (SHB) effect is analyzed theoretically. The stability of the laser structure is evaluated using the flatness parameter $F$. It is shown that the LC-DFB lasers are stable in a wide range of different values of ridge width and amplitude grating. The stability is sustained with increasing injection current. The results show that the flatness parameter is minimal for certain values of the amplitude grating. The numerical calculations have been done by coupled wave and carrier rate equations on the basis of the transfer matrix method (TMM).
\end{abstract}

Keywords-component; laterally coupled distributed feedback laser; spatial hole burning; rate equation; coupled wave equation; transfer matrix method

\section{INTRODUCTION}

The semiconductor DFB lasers, due to their low threshold current, narrow line width and single mode capability, are considered as an important optical source in communications systems [1]. Conventional DFB lasers with coupling refractive index with end facets coated with anti reflection, AR, are not single-moded and have two symmetrical modes relative to Bragg mode that appear simultaneously in the laser output [2]. One way for having a single mode in AR-DFB laser is by employing an equivalent phase shift at the center of the grating in the structure $[3,4]$. This laser, which is also named QWS DFB, has high efficiency but is sensitive to the reflection at the facets [5]. Another major problem of this structure is its high non-uniformity in the distribution of photons in the direction along the length of the cavity which in turn results in undesirable Spatial Hole Burning (SHB). This is because of the effect of phase shift which causes instability in the laser [6]. On the other hand, LC-DFB lasers use a lithographic fabrication step to define the distributed feedback grating, avoiding subsequent regrowth. By avoiding regrowth steps, these lasers may be monolithically integrated with other optoelectronic devices [7, 8].This makes this type of lasers not only easier to fabricate but also less expensive.

This article reports, for the first time, on the stability of LC-DFB lasers against SHB, considering both the structural parameters and alterations of the density of carriers and photons along the length of the cavity structure and its dependence to the current above threshold conditions.

The theoretical analysis is carried out by simultaneous solving both the coupled wave and rate equations using the transfer matrix method. In section 2, theory and the physical model are explained. Section 3 explains the results and section 4 summarizes the conclusions.

\section{THEORETICAL AND PHYSICAL MODEL}

Figure 1 illustrates a simple design of a Five-layered LCDFB laser and its features. In this figure, $\mathrm{d}, \mathrm{h}$ and $\mathrm{t}$ are the active, buffer and wave guide layer thicknesses respectively. A clearer view of a LC-DFB laser is depicted in Figure 2. As shown, there is a grating over the waveguide at both sides of the central area which in turn makes distributed reflection in the laser.

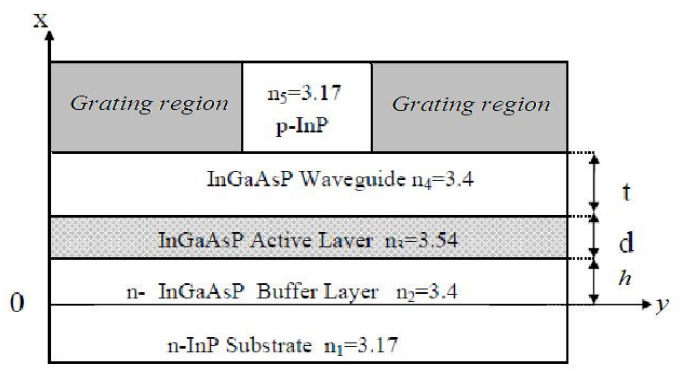

Fig. 1. Simple design of a DFB laser structure with lateral coupling

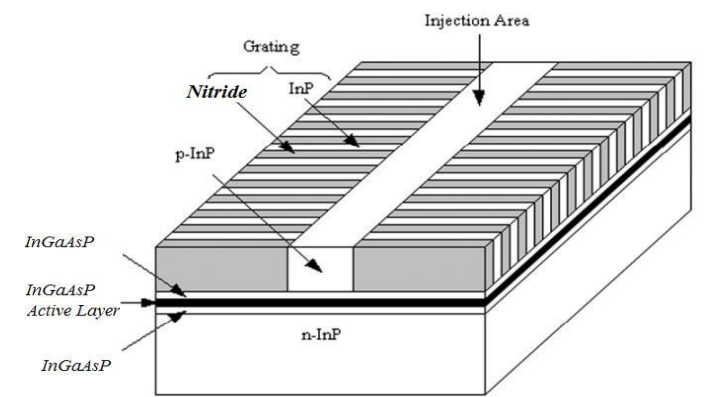

Fig. 2. The schematic of laterally-coupled distributed feedback laser [10]. 
We consider the direction of the electric field along y-axis, TE mode. Electromagnetic waves propagate both in positive and negative directions along the Z-axis in the cavity. The modified coupled-mode equations, first described by Streifer, are given by [9]:

$$
\left\{\begin{array}{c}
\frac{d A(z)}{d z}-j \delta A(z)=j \kappa B(z) \\
-\frac{d B(z)}{d z}-j \delta A(z)=j \kappa A(z)
\end{array}\right.
$$

Where A and B are the forward and backward propagating complex amplitudes of the field respectively; $\kappa$ is the coupling coefficient between forward and backward propagating modes; $\delta=\beta-\beta_{0}$ is the Bragg wavelength detuning; $\beta_{0}=m \pi / \Lambda$ is the propagation constant, in which $\mathrm{m}$ is the grating order.

Carrier density in the active region follows the rate equation. This equation for single-moded DFB laser is as follows [11]:

$$
\begin{aligned}
& \frac{\partial N(z, t)}{\partial t}=\frac{J}{q d} \\
& +\left[A+B N(z, t)+C N(z, t)^{2}\right]-G(z, t) S(z, t)
\end{aligned}
$$

Where $N(z, t)$ and $S(z, t)$ are carrier and photon densities respectively, $J$ is input current density; $q$ is the electronic charge; $d$ is the active layer thickness; $A, B$ and $C$ are the nonradiative, spontaneous and auger rates respectively. Photon density at every point is proportional to the sum of the square of forward and backward traveling wave amplitudes. In (2), stimulated emission rate $G$ is equal to:

$$
G(z, t)=v_{g} g_{m a t}(z, t)
$$

In which $v_{g}$ is the group velocity and $g_{\text {mat }}(z, t)$ has linear relationship with carrier density, as shown in (4) below [12]:

$$
g_{\text {mat }}(z, t)=a_{n}\left[N(z, t)-N_{t}\right]
$$

Where $N_{t}$ is the carrier density in transparency conditions and $a_{n}$ is the differential gain coefficient. The refractive index changes in the active region in the following equation depend on carrier density [13]:

$$
\Delta n(z, t)=-\frac{\alpha_{H} \lambda_{0} a_{n} \Delta N(z, t)}{4 \pi}
$$

In which $\alpha_{H}$ is the line width coefficient and $\Delta N$ is the carrier density fluctuation with relation to the threshold conditions. Also $\delta$ above threshold conditions considering the changes in the refractive index is as follows:

$$
\delta(z, t)=\frac{2 \pi}{\lambda_{0}}\left(n_{e f f}+\Gamma \Delta n\right)-\frac{n \pi}{\Lambda}
$$

Where $\Lambda$ is the grating period and $n_{\text {eff }}$ is the effective refractive index. Equation (7) shows the relationship between $g_{\text {mat }}$ and the modal gain, $\alpha_{\bmod a l}[14]$ :

$$
g_{m a t}=\frac{2 \alpha_{m}+\alpha_{\bmod a l}}{\Gamma_{a c}}
$$

In this equation $\Gamma_{a c}$ is the optical confinement factor in the active layer and $\alpha_{\bmod a l}$ is the average internal loss. The values of $\alpha_{m}$ and $\delta$ are obtained by using the boundary conditions $R(0)=r_{1} S(0)$ and $S(L)=r_{2} R(L) . r_{1}$ and $r_{2}$ are the reflection coefficients of the mirrors that are assumed to be zero here. The stability of the laser against SHB effect is measured using the flatness parameter $F$, which is defined as follows:

$$
F=\frac{1}{L} \int_{0}^{L}\left(\frac{I(z)}{I_{a v g}}-1\right)^{2} d z
$$

In (8), I(z) is the optical field intensity along the cavity length and $I_{a v g}$ is the average value of $\mathrm{I}(\mathrm{z})$.

$$
I(z)=\left|E_{R}(z)\right|^{2}+\left|E_{S}(z)\right|^{2}
$$

The smaller the value of $\mathrm{F}$, the more uniform is the distribution of light field along the cavity and less is the possibility of the existence of SHB. Experimentally it has been shown that for sustainable performance of single mode DFB laser F should be less than 0.05 [6]. Analysis is based on solving both the coupled wave equations (1) and rate equation (4) simultaneously. The method employed based upon TMM $[15,16]$. 
In this method, the cavity length is divided in to 200 equal parts and in every part, different parameters of the laser including coupling coefficient, carrier density, refractive index and density of photons are assumed to be constant. But their values are different from one part to the next. Ref. [10] has been used as the bench mark for our calculations for threshold conditions to the first order grating.

The values of the parameters used in our calculations are presented in Table 1. In LC-DFB laser structure by choosing a suitable ridge height and width and also an appropriate input current, we will obtain a structure with minimum flatness parameter above the threshold conditions.

TABLE I. THE VALUES OF THE PARAMETERS

\begin{tabular}{|c|c|}
\hline Parameters & Values \\
\hline Cavity length $(\mathrm{L})$ & $500 \mu \mathrm{m}$ \\
\hline Laser wavelength $\left(\lambda_{0}\right)$ & $1.5 \mu \mathrm{m}$ \\
\hline Buffer layer thickness $(\mathrm{h})$ & $150 \mathrm{~nm}$ \\
\hline Waveguide layer thickness $(\mathrm{t})$ & $150 \mathrm{~nm}$ \\
\hline Internal dissipation of the active layer $\left(\alpha_{\text {int }}\right)$ & $20 \mathrm{~cm}^{-1}$ \\
\hline Improved line width coefficient $\left(\alpha_{0}\right)$ & 6 \\
\hline Nonradiative combination rate $(\mathrm{A})$ & $10^{8} \mathrm{~s}^{-1}$ \\
\hline Spontaneous recombination rates $(\mathrm{B})$ & $8 \times 10^{-11} \mathrm{~cm}^{3} \mathrm{~s}^{-1}$ \\
\hline Auger recombination rates $(\mathrm{C})$ & $7.5 \times 10^{-29} \mathrm{~cm}^{6} \mathrm{~s}^{-1}$ \\
\hline
\end{tabular}

For our modeling purposes, first we have employed the effective refractive index method [17], and have calculated the changes both in the X-and Y-directions. Ultimately, we then used the TMM only along the Z-direction. The above procedure makes our simulation in all 3 directions. We have considered the effective refractive index under the ridge and the area around it. We then used our effective index calculations to compute the lateral coupling coefficient on both sides of the ridge.

\section{EVALUATION OF RESULTS}

In Figure 3, the flatness parameter in terms of the grating amplitude, a, for three different values of the ridge width, W, $(1.5,2,2.5 \mu \mathrm{m})$ is depicted and as shown, the flatness parameter for certain values of the grating amplitude is minimum.

The corresponding grating amplitudes are known as the critical amplitudes, $a_{c}$. This critical value depends on the width of the ridge. For example, when $\mathrm{W}=1.5 \mu \mathrm{m}$, the critical amplitude is approximately $0.04 \mu \mathrm{m}$. Also the critical amplitude for ridge widths of $2 \mu \mathrm{m}$ and $2.5 \mu \mathrm{m}$ are $0.04 \mu \mathrm{m}$ and $0.07 \mu \mathrm{m}$ respectively. Figures 5 and 6 show the same trend for different values of $\mathrm{W}$ and $\mathrm{a}_{\mathrm{c}}$.

It is important to note that the behavior of the photon and carrier densities for grating amplitudes, smaller and/or greater values than that of the corresponding critical amplitude are different. Figure 4 is for $\mathrm{W}=1.5 \mu \mathrm{m}$ and $a_{c}=0.04 \mu \mathrm{m}$.

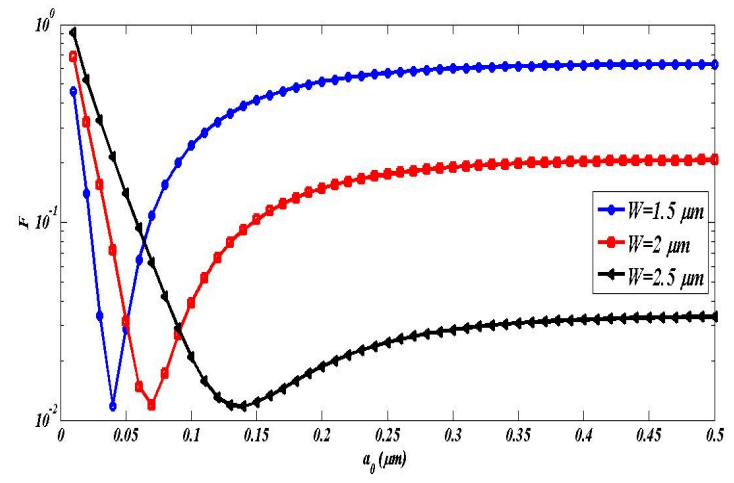

Fig. 3. Flatness changes Vs grating amplitude
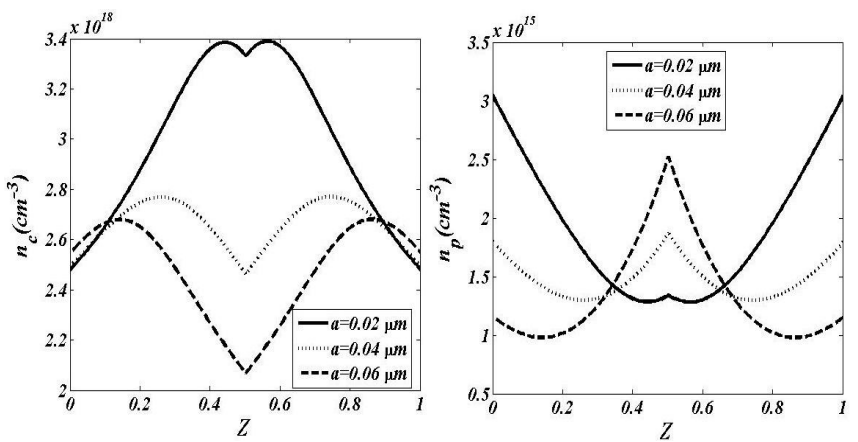

Fig. 4. Shows $n_{p}$ and $n_{c}$ changes along the cavity length for $a_{c}=0.04 \mu m$ and $\mathrm{W}=1.5 \mu m$
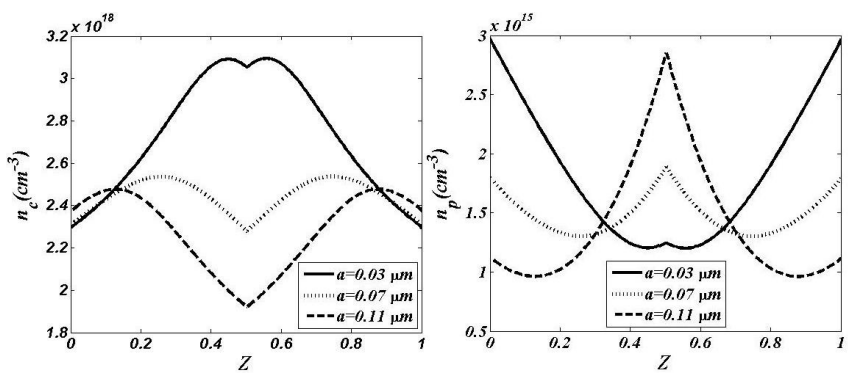

Fig. 5. Shows $n_{p}$ and $n_{\mathrm{c}}$ changes along the cavity length for $\mathrm{W}=2 \mu \mathrm{m}$ and $a_{c}=0.07 \mu \mathrm{m}$.
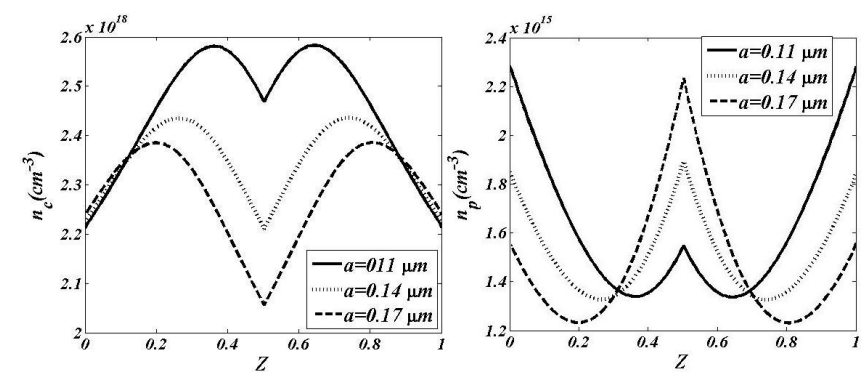

Fig. 6. Shows $n_{p}$ and $n_{c}$ changes along the cavity length for $\mathrm{W}=2.5 \mu \mathrm{m}, a_{c}=0.14 \mu \mathrm{m}$ 
As shown, when $a\left\langle a_{c}\right.$, photons density $\mathrm{n}_{\mathrm{p}}$ at the two ends of the laser is increased and when $a>a_{c}$ photons density at the center of structure has its maximum value. When $a=a_{c}$, the photon density has its least changes as compared to other values of $a$. This is also true for the carrier density, $n_{c}$ in the structure.

This behavior of LC-DFB laser shows that in critical amplitude regime, SHB has the least effect on the laser. Figures 7-9 illustrate the flatness parameters in terms of the input current for $W=1.5 \mu \mathrm{m}, W=2 \mu \mathrm{m}, W=2.5 \mu \mathrm{m}$ respectively. It can be seen from the figures that the minimum flatness parameter is achieved at the critical amplitude, $a_{c}$ even though the input current is on the rise.

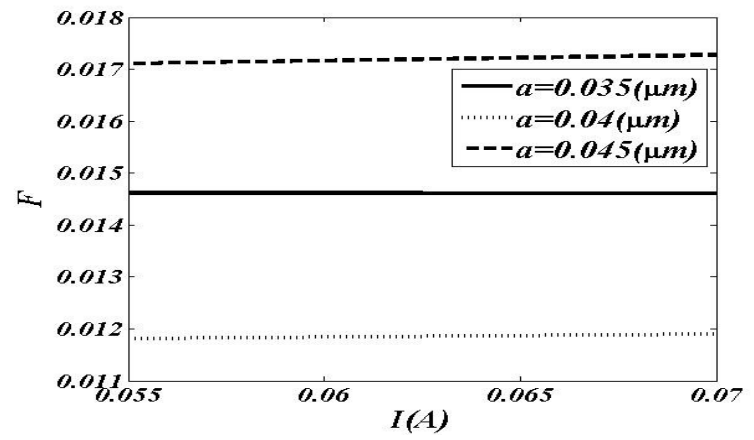

Fig. 7. Flatness parameter vs. input current for different value of grating amplitudes with $\mathrm{W}=1.5 \mu \mathrm{m}$.

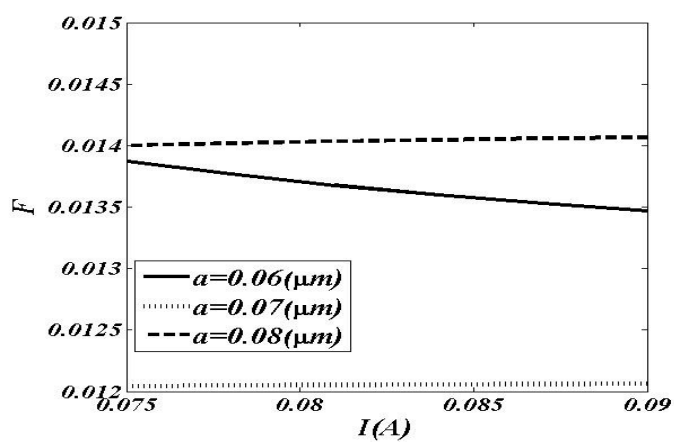

Fig. 8. Flatness parameter vs. input current for different value of grating amplitudes with $\mathrm{W}=2 \mu \mathrm{m}$.

Figure 10 shows the changes of flatness parameter in terms of varying input current for different grating amplitudes. It is shown that the structure relating to the critical amplitude, has the minimum flatness parameter F. Also it is important to note that the structure with critical parameter $a_{c}$ has the minimum changes of $\mathrm{F}$ with changing current. This fact indicates that the best stability against SHB is achieved with those structures with critical amplitudes.

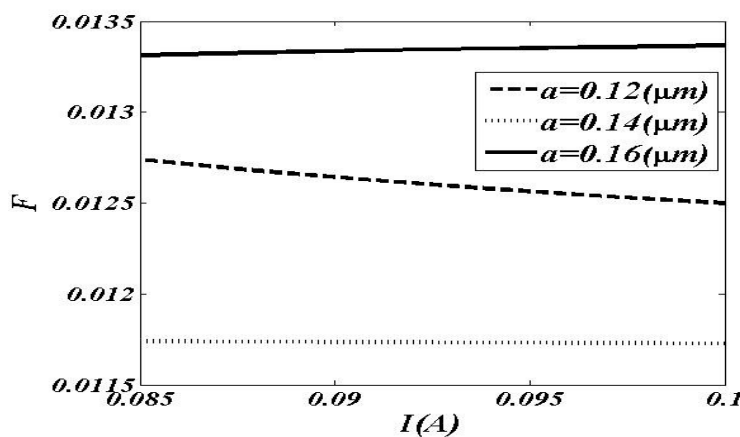

Fig. 9. Flatness parameter Vs. input current for different value of grating amplitudes with and $\mathrm{W}=2.5 \mu \mathrm{m}$.

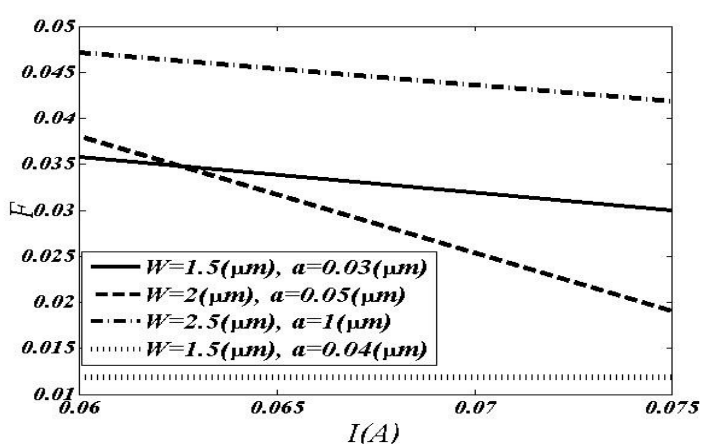

Fig. 10. Changes of flatness parameter in terms of varying input current for different grating amplitudes

\section{CONCLUSION}

Flatness parameters in LC-DFB lasers in and above threshold conditions are analyzed theoretically. Calculations show that the $\mathrm{F}$ parameter has the least value for different structures at critical amplitude and at points close to the corresponding critical amplitude is less than 0.05. Considering the changes of both carrier and photon densities along the cavity and also above the threshold, shows that the minimum density changes occur for structures with critical amplitudes. Also changes in $\mathrm{F}$ for different structures at critical amplitudes, above threshold conditions with increasing input current, are negligible. These results show the high stability of LC-DFB lasers against SHB effect with increasing input current.

\section{REFERENCES}

[1] K. Nosu, K. Iwashita, "A consideration of factors affecting future coherent lightwave communication", J. Lightwave Technol. Vol. 6, No. 5, pp. 686-694, 1988

[2] H. Kogelnik, C. V. Shank, "Coupled-wave theory of distributed feedback lasers”, J. Appl. Phys., Vol. 43, No. 5, pp. 2327-2335, 1972

[3] K. Utaka, S. Akiba, K. Saki, Y. Matsushima, “ $\lambda / 4$-shifted InGaAsP DFB laser,” IEE J. Quantum Electron., Vol. 22, No. 7, pp. 1042-1051, 1986

[4] H. Ghafouri-Shiraz, B. S. K. Lo, Distributed feedback laser diodes: principles and physical modelling, John-Wiley \& Son, 1996

[5] J. Kinoshita, K. Matsumoto, "Yield analysis of SML DFB lasers with an axially-flattened internal field", IEE J. Quanrum Electron., Vol. 25, pp 1324-1332, 1989 
[6] H. Soda, Y. Kotaki, H. Sudo, H Ishikwa, S. Yamakoshi, H. Imai, "Stability in single longitudinal mode operation in single longitudinal mode operation in GaInAsP/Inp phase-adjusted DFB lasers", IEEE J . Quantum Electron., Vol. 23, No. 6, pp. 804-814, 1987

[7] R. Millett, K. Hinzer, T. Hall, H. Schriemer "Simulation Analysis of Higher Order Laterally-Coupled Distributed Feedback Lasers”, IEEE J. Quantum Electron. Vol. 44, No. 12, pp. 1145-1151, 2008.

[8] R. Millett, H. Schriemer, T. Hall, K. Hinzer, "Properties of LaterallyCoupled Distributed Feedback Lasers with Higher Order Gratings," NUSOD '08, International Conference on Numerical Simulation of Optoelectronic Devices, pp. 13-14, United Kingdom, 2008

[9] W. Streifer, D. R. Scifres, R. D. Burnham, "Coupled wave analysis of DFB and DBR lasers", IEEE J. Quantum Electron., Vol. 13, No. 4, pp. 134-141, 1977.

[10] J. Wang, J. Tian, P. Cai, B. Xiong C. Sun, Y. Luo, " $1.55-\mu \mathrm{m}$ AlGaInAs-InP Laterally Coupled Distributed Feedback Laser”, IEEE Photonics Technology Letters, Vol. 17, No. 7, pp. 1372-1374, 2005.

[11] G. P. Agrawal, A. K. Dutta, Semiconductor lasers, Van Nostrand Reinhold, 1966
[12] M. Osinsky, M. J. Adams, "Gain spectra of quaternary semiconductor", IEE Proc. In Solid-State and Electron Devices, Vol. 129, No. 6, pp. 229236, 1982

[13] S. F. Yu, "Double-tapered-Waveguide distributed feedback lassers for high-power single mode operation", IEE J. Quantum Electron., Vol. 33, No. 1, pp. 71-80, 1997

[14] K. David, J. Buus, G. Morthier, R. Baest, "Coupling coefficients in gain coupled DFB lasers: Inherent compromise between coupling strength and loss", IEEE Photonics Technology Letters, Vol. 3, No. 5, pp. 439441,1991

[15] F. Shahshahani, V. Ahmadi, "Analysis of relative intensity noise in tapered grating QWS-DFB laser diodes by using three rate equations model", Solid-State Electronics, Vol. 52, No. 6, pp. 857-862, 2008

[16] T. Makino, "Transfer-matrix formulation of spontaneous emission noise of DFB semiconductor lasers", J. Lightwave Technol., Vol. 9, No. 1, pp. 84-91, 1991

[17] G. Agrawal, Fiber-optic communication system. John Wiley \& Sons, 1992 